\title{
CHLOROPHYTA FILAMENTOSAS DO MUNICÍPIO DE CÁCERES E ARREDORES, MATO GROSSO, BRASIL: UMA CONTRIBUIÇÃO AO SEU CONHECIMENTO'
}

\author{
Izabel Cristina Alves Dias²
}

Recebida em 3-2-89. Aceito em 7-12-89.

RESUMO - São descritas, comentadas e ilustradas 12 espécies de Chlorophyta filamentosas coletadas durante a II Expedição do Programa Polonoroeste/Conselho Nacional de Desenvolvimento Científico e Tecnológico/Museu Nacional da Universidade Federal do Rio de Janeiro à região de Cáceres e arredores, Mato Grosso, Brasil. Do total de espécies apresentadas neste trabalho nove constituem primeiras citações de ocorrência no Estado de Mato Grosso e seis no Brasil.

Palavras-chave: Algas, Chlorophyta filamentosas, Mato Grosso, Brasil.

ABSTRACT - Twelve species of filamentous Chlorophyta from the municipality of Cáceres and surroundings, state of Mato Grosso, Brazil, are described, illustrated, and discussed. Nine species were documented for the first time for Mato Grosso, and six for the Brazilian territory.

Key words: Algae, filamentous Chlorophyta, Mato Grosso, Brazil.

\section{Introdução}

O Programa Polonoroeste/Conselho Nacional de Desenvolvimento Científico e Tecnologico/Museu Nacional da Universidade Federal do Rio de Janeiro permitiu o estudo da biota na área sob influência da BR-364 (Cuiabá-Porto Velho). Parte da ficoflora da região está documentada em Dias (1986) e Menezes

1 - Parte do subprojeto "Estudos Botânicos da área sob influência da BR-364 (Cuiabá-Porto Velho)": Programa Polonoroeste, Conselho Nacional de Desenvolvimento Científico e Tecnológico/Museu Nacional da Universidade Federal do Rio de Janeiro.

2 - Departamento de Botânica, Museu Nacional, Quinta da Boa Vista, São Cristóvão, 20942, Rio de Janeiro, RJ. 
(1986) para a Chapada dos Guimarães e arredores (I Expedição) e Menezes \& Fernandes (1987) para Cáceres e arredores (II Expedição). Com o presente trabalho, ampliamos o conhecimento da ficoflórula brasileira e, em especial, da região investigada, com a análise das amostras coletadas durante a II Expedição do Programa.

\section{Material e Métodos}

A área estudada abrangeu os munić́pios de Cáceres, Mirassol d'Oeste, Rio Branco, Quatro Marcos, Salto do Céu e Barra do Bugres, no período de novembro a dezembro de 1984 (fig. 1).

Foram analisadas 12 amostras coletadas em 56 diferentes corpos e cursos d'água. A metodologia de coleta e as técnicas de preservação do material encontram-se em Dias (1986) e a descrição detalhada da área de coleta em Menezes \& Fernandes (1987).

Do total de amostras coletadas e examinadas, apenas as 22 abaixo discriminadas apresentaram representantes de Chlorophyta filamentosas.

1 - Município de Cáceres, Porto Esperidião, Córrego Carrapato, Fazenda Pantanalzinho, aderido à vegetação marginal, col. V. Huszar, 22.XI.1984 (R 160654).

2 - Munićpio de Cáceres, Porto Esperidião, Fazenda Pantanalzinho, Baáa do Campo, recolhimento de macrófita aquática, col. V. Huszar, 23.XI.1984 (R 160655).

3 - Munić́pio de Cáceres, Porto Esperidião, Fazenda Três Meninas, lagoa ao fundo do silo da fazenda, recolhimento de massas de algas filamentosas junto às macrófitas aquáticas, col. V. Huszar, 25.XI.1984 (R 155576).

4 - Município de Cáceres, Porto Esperidião, Fazenda do Sr. Carlos Emílio, charco próximo à margem direita do Rio Vermelho, recolhimento de algas filamentosas, col. V. Huszar, 27.XI.1984 (R 160656).

5 - Município Quatro Marcos, Fazenda Favorita, açude, plâncton de rede, col. V. Huszar, 29.XI.1984 (R 160657).

6 - Município de Quatro Marcos, Fazenda Favorita, córrego próximo à fazenda (estrada Mirassol d'Oeste - Santa Fé), recolhimento de algas filamentosas, col. V. Huszar, 29.XI.1984 (R 155583).

7 - Munić́pio de Mirassol d'Oeste, Serra do Caeté, empoçado em pedreira de calcário, recolhimento de algas filamentosas, col. V. Huszar, 29.XI.1984 (R 160658).

8 - Município de Cáceres, BR-174 (Cáceres - Pontes e Lacerda), km 45, alagado do Córrego Caramujo, recolhimento de macrófitas, col. V. Huszar, 02.XII.1984 (R 160659).

9 - Município de Cáceres, Córrego Sangradouro, recolhimento de algas filamentosas, col. V. Huszar, 02.XII.1984 (R 160660). 
10 - Município de Barra do Bugres, Fazenda Bocaína, cachoeira do Córrego Três Ribeirões, aderido às macrófitas, col. V. Huszar, 04.XII.1984 (R 160661).

11 - Município de Cáceres, Fazenda Santo Antônio, Baía do Espinhal Grande, meandro divagante do Rio Paraguai, passagem de frasco e recolhimento de macrófitas, col. V. Huszar, 05.XII.1984 (R 155591).

12 - Munić́pio de Barra do Bugres, Nova Fernandópolis, Alto do Córrego do Hildo, recolhimento de algas filamentosas, col. A.C. Domingos, 30.X.1984 (R 160662).

13 - Município de Barra do Bugres, Nova Fernandópolis, Rio das Antas (estrada Sant'Ana do Oeste - Cáceres), recolhimento de algas filamentosas, col. A.C. Domingos, 19.XI.1984 (R 160663).

14 - Município de Barra do Bugres, Nova Fernandópolis, Córrego da Água Boa (estrada Sant'Ana do Oeste - Cáceres), recolhimento de algas filamentosas, col. A.C. Domingos, 19.XI.1984 (R 160664).

15 - Município de Salto do Céu, Córrego Limpo (MT-246, estrada Progresso - Salto do Céu), recolhimento de algas filamentosas, col. A.C. Domingos, 13.XI.1984 (R 160665).

16 - Munić́pio de Salto do Céu, Rio Caio, recolhimento de algas filamentosas, col. A.C. Domingos, 30.X.1984 (R 160666).

17 - Município de Barra do Bugres, Córrego dos Patos, recolhimento de algas filamentosas, col. A.C. Domingos, 20.X.1984 (R 160667).

18 - Município de Barra do Bugres, estrada Barra do Bugres - Nova Fernandópolis, Rio Branco, recolhimento de algas filamentosas, col. A.C. Domingos, 14.XI.1984 (R 160668).

19 - Munić́pio de Barra do Bugres, Rio Rico, recolhimento de algas filamentosas e macrófitas, col. A.C. Domingos, 08.XI.1984 (R 160669).

20 - Munić́pio de Barra do Bugres, Nova Fernandópolis, estrada Barra do Bugres - Nova Fernandópolis, riozinho, recolhimento de algas filamentosas, col. A.C. Domingos, 21.XI. 1984 (R 160670).

21 - Munić́pio de Barra do Bugres, Nova Fernandópolis, Rio Claro, recolhimento de algas filamentosas, col. A.C. Domingos, 22.XI.1984 (R 160671).

22 - Município de Barra do Bugres, Nova Fernandópolis, Rio Sepotuba, recolhimento de macrófitas aquáticas e algas filamentosas, col. A.C. Domingos, 18.XI.1984 (R 160672).

\section{Resultados e Discussão}

Das 128 amostras analisadas, 22 apresentaram representantes de Chlorophyta filamentosas e possibilitaram a identificação de 12 espécies distriburdas pelos gêneros Mougeotia C. Agardh, Spirogyra Link e Zygnema C. Agardh (Zygnemaphyceae); Oedogonium Link (Oedogoniaceae); Microspora Thuret 
(Microsporaceae); Stigeoclonium Kützing (Chaetophoraceae); e Schizomeris Kützing (Schizomeridaceae).

Do total de espécies descritas, comentadas e ilustradas neste trabalho, nove são primeiras citações de ocorrência para o estado de Mato Grosso, à exceção de Spirogyra neglecta (Hassall) Kützing (Dias, 1986), Stigeoclonium tenue (Agardh) Kützing var. tenue (Schmidle, 1901), Oedogonium reinschii Roy (Borge, 1925) e Microspora willeana Lagerheim (Prescotty, 1957).

Spirogyra fennica Cedercreutz, Spirogyra minutifossa Jao, Zygnema oveidanum Transeau, Zygnema insigne (Hassal) Kützing, Microspora tumidula Hazen e Stigeoclonium farctum Berthold são primeiras citações de ocorrência no Brasil.

\section{ZYGNEMACEAE}

Mougeotia abnormis Kisselev (fig. 2).

Bull. Bur. Applied Ichthyol. 5:301. pl. 1, fig. 6. 1927.

Células vegetativas $15,0-18,0 \times 155,0-180,0 \mu \mathrm{m}$, cloroplastídio com 5-8 pirenóides em série única; conjugação escariforme; zigósporos globosos a triangular-elipsóides, ocupando metade do tubo e metade do gametângio receptivo, 39,0-42,5X32,0-36,5 $\mu \mathrm{m}$; mesరsporos lisos, castanhos.

Material examinado: R155576.

Spirogyra fennica Cedercreutz (fig. 3-4).

Acta Soc. Fauna Flora Fenn. 55(2):4. 1924.

Células vegetativas $15,5-18,0 \times 72,0-100,0 \mu \mathrm{m}$, septos planos, 1 cloroplastídio; conjugação escalariforme, tubos formados por ambos gametângios; células férteis infladas, 27,0-32,0 $\mu \mathrm{m}$; zigósporos elipsóides, 20,0-23,0X39,0-47,5 $\mu \mathrm{m}$; mesósporos espessos, lisos, castanho-amarelados.

Material examinado: R160665 e R160666.

Spirogyra minutifossa Jao (fig. 5-6).

Trans. Am. microsc. Soc. 54:3-4, pl. 1, fig. 51935.

Células vegetativas 13,0-16,0X65,5-95,0 $\mu \mathrm{m}$, septos planos, 1 cloroplastídio; conjugação escalariforme, tubos formados por ambos gametângios; células férteis fusiformes, infladas, 21,0-24,0 $\mu \mathrm{m}$; zigósporos elipsóides, $19,0-25,0 \times 35,0-38,5 \mu \mathrm{m}$; mesósporos com estrias irregularmente retorcidas, amarelados.

Material examinado: R155576.

$\mathrm{O}$ material examinado apresentou as células vegetativas e os zigósporos ligeiramente mais estreitas que o mencionado na descrição original, concordando, entretanto, nas demais características.

Spirogyra neglecta (Hassall) Kützing (fig. 7).

Spec. Algar. 441. 1849. 
Células vegetativas $64,0-71,5 \mathrm{X} 210,0-275,0 \mu \mathrm{m}$, septos planos, $3-4$ cloroplastídios; conjugação escalariforme, tubos formados por ambos gametângios; células férteis cilíndricas; zigósporos elipsóides, pólos arredondados, 53,5-61,0X78,5-90,0 $\mu \mathrm{m}$; mesósporos espessos, lisos, castanho-amarelados.

Material examinado: R160659.

Zygnema oveidanum Transeau (fig. 10-11).

Trans. Am. Microsc. Soc. 53:208, pl. 16, fig. 1. 1934.

Células vegetativas 10,0-12,5X40,0-65,0 $\mu \mathrm{m}$; conjugação escalariforme; zigósporos globosos formados nos tubos de conjugação, 14,0-16,5X16,0-25,0 $\mu \mathrm{m}$; mesósporos pontuados (ca. $1 \mu \mathrm{m}$ diâm. cada), castanhos.

Material examinado: R155576.

Zygnema insigne (Hassall) Kützing (fig. 8-9).

Spec. Algar. 444. 1849.

Células vegetativas 25,0-29,0X46,0-52,5 $\mu \mathrm{m}$; conjugação escalariforme; células férteis alargadas, 39,5-43,5 $\mu \mathrm{m}$; zigósporos globosos a subglobosos formados nos gametângios, 27,5-35,0X28,0-35,5 $\mu \mathrm{m}$; mesósporos lisos, castanho-amarelados.

Material examinado: R 160654.

\section{OEDOGONIACEAE}

Oedogonium reinschii Roy (fig. 12).

In: Cooke, Brit. freshw. algae 160, pl. 57, fig. 23. 1884.

Células vegetativas hexagonais, $6,0-8,5 \times 12,0-15,0 \mu \mathrm{m}$, cloroplastídio indistinto. Material esterril.

Material examinado: R160670.

\section{MICROSPORACEAE}

Microspora tumidula Hazen (fig. 13-16).

Mem. Torrey Bot. Club 11(2): 177, pl. 24, fig. 8-11. 1902.

Células vegetativas freqüentemente cilíndricas, ligeiramente constritas nos septos, 7,5-9,0X10,0-18,5 $\mu \mathrm{m}$; peças em $\mathrm{H}$ visf́veis em condições vegetativas; cloroplastídio perfurado cobrindo larga porção da parede celular; aplanósporos arredondados, 5,5-7,5 $\mu \mathrm{m}$ de diâmetro.

Material examinado: R160656.

Microspora willeana Lagerheim (fig. 17-20).

Ber. Dd. bot. Ges. 5:414. 1887.

Células vegetativas cilf́ndricas ou ligeiramente quadrangulares, algumas vezes constritas nos septos, 10,0-13,5X11,0-15,0 $\mu \mathrm{m}$, peças em $\mathrm{H}$ pouco visí- 
veis em condições vegetativas; cloroplastídio freqüentemente em placa perfurada cobrindo apenas as paredes laterais das células; acinetos 10,0-12,0 $\mu \mathrm{m}$ de largura; aplanósporos 1 ou 2 por células, 5,5-7,5 $\mu \mathrm{m}$ de diâmetro, raros.

Material examinado: R160665 e R160667.

Esta espécie é citada na literatura como muito próxima a Microspora flocosa (Vaucher) Thuret. Dias (1985) comenta a variabilidade métrica do diâmetro das células com base em Hazen (1902), Prescott (1975) e Ramanathan (1964) e conclui que, embora próximas, as espécies em questão devem ser consideradas isoladamente. A maior largura dos acinetos em Microspora flocosa é tamberm forte caráter distintivo.

Os raros aplanósporos encontrados nos exemplares estudados apresentaram diâmetro um tanto menor que o mencionado na literatura.

\section{CHAETOPHORACEAE}

Stigeoclonium farctum Berthold (fig. 21-22).

Nova Acta Leopoldina 40(5): 201, pl. 2, fig. 1-5. 1878.

Células vegetativas do sistema ereto cilíndricas, 6,5-9,5X19,0-33,0 $\mu \mathrm{m}$; ramificações freqüentemente alternadas e dicotômicas, com ápices pontiagudos; cloroplastídio parietal, em placa; sistema basal formado por células aproximadamente isodiamétricas formando uma base pseudoparenquimatosa.

Material examinado: R160657.

Stigeoclonium tenue (C. Agardh) Kützing var. tenue (fig. 23-24).

Phycol. gener. 253. 1843.

Células vegetativas do sistema ereto cilíndricas, ligeiramente constritas, $11,0-14,5 \times 28,0-43,0 \mu \mathrm{m}$; ramificações simples e opostas a partir de células curtas do eixo principal, ápices dos ramos freqüentemente providos de formações alongadas e hialinas ou apenas pontiagudas; cloroplastídio preenchendo as células mais curtas do eixo principal e ocupando apenas a porção mediana nas mais longas; sistema basal não observado.

Material examinado R155583.

\section{SCHIZOMERIDACEAE}

Schizomeris leibleinii Kützing (fig. 25-26).

Phycol. gener. 247. 1843.

Células vegetativas da região basal do talo cilíndricas, $25,0-48, \mu \mathrm{m}$ de diâmetro; células da região distal do talo geralmente cúbicas, 13,0-18,0 $\mu \mathrm{m}$ de lado; região basal unisseriada, cloroplastídio parietal formando uma faixa na região mediana da célula; região distal multisseriada, cloroplastídio reticulado ou de formato irregular formando uma massa densa; pirenбides presentes.

Material examinado: R155583.

Dias (1985) comenta, com base em material coletado no estado do Rio de 
Janeiro, a problemática envolvendo a posição taxonômica do gênero e a validade de Schizomeris irregularis Frists \& Rich, uma espécie descrita para a África e que, em nossa opinião, não está bem caracterizada.

Campbell \& Sarafis (1972) comentam estudos sobre polimorfismo, reprodução, estrutura celular e ultra-estrutura em Stigeoclonium tenue e Schizomeris, a partir da observação destes táxons em épocas alternadas em um determinado lago na Nova Zelândia. Os resultados indicaram que a forma Schizomeris foi adotada por Stigeoclonium sob condições desfavoráveis. Pereira \& Parra (1984) descrevem Stigeoclonium tenue para o Chile e comentam que, em parte da amostra desenvolvida em cultura, foram observados estágios palmelóides e filamentos do tipo Schizomeris.

No presente artigo, optamos pela identificação de $S$. tenue e Sleibleinii segundo as características padronizadas para cada espécie, tendo em vista o fato de termos analisado amostras preservadas e a impossibilidade imediata de mantermos um acompanhamento na natureza e em cultivo para obtermos nossos próprios resultados.

Vale ressaltar que Islam (1963) e Cox \& Bold (1966), em artigos especializados sobre Stigeoclonium, consideraram $S$. tenue uma espécie altamente polimórfica.

Acreditamos que as observações acima com relação a estes dois táxons merecem ainda maiores estudos por parte dos especialistas.

\section{Referências Bibliograficas}

BERTHOLD, G. 1878. Untersuchungen über Verzwigung einiger Süsswaseralgen. Nova Acta Leopoldina, Leipzig, 40 (5): 169-230.

BORGE, O. 1925. Die von Dr. F.C. Hoehne während der Expedition Roosevelt-Rondon gesamelten Süsswaseralgen. Ark. Bot., Uppsala, 19 (17): 1-56.

CAMPBELL, E. \& SARAFIS, V. 1972. Schizomeris a growth form of Stigeoclonium tenue (Chlorophyta, Chaetophora). J. Phycol., Lawrence, 8 (3): 276-283.

CEDERCREUTZ, C. 1924. Finnländische Zygnemalen. Acta Soc. Fauna Flora Fenn., Helsingforsiae, 55 (2): 1-7.

COX, E.R. \& BOLD, H.C. 1966. Phycologial studies. VII. Taxonomic investigations of Stigeoclonium. Austin, University of Texas, 167p. (Publ. no 6618).

DIAS, I.C.A. 1985. Chlorophyta filamentosas da Lagoa de Juturnaiba, Araruama, Rio de Janeiro. Revta Brasil. Bot., São Paulo, 8: 93-95.

DIAS, I.C.A. 1986. Zygnemaceae (Zygnemaphyceae) da Chapada dos Guimarães e arredores, Mato Grosso, Brasil: uma contribuição ao seu conhecimento. Rickia, São Paulo, 13: 69-75.

HAZEN, T.E. 1902. The Ulotricaceae and Chaetophoraceae of United States. Mem Torrey Bot. Club, New York, 11 (2): 1 - 250

ISLAM NURUL, A.K.M. 1963. A revision of the genera Stigeoclonium. Beih. Nova Hedwigia, Weinheim, 10.164 p.

JAO, C.C. 1935. New Zygnemataceae from Woods Hole. Trans. Am. microsc. Soc., Lancaster, 54: 1-7. 
KISSELEV, J.A. 1927. Zur Kenntnis der Algen des Aralses. Bull. Bur. Applied Ichthyol., Leningrad, 5: 301.

KÜTZING, F.T. 1843. Phycologia generalis. Leipzig, Brockhaus, 458 p.

KÜTZING, F.T. 1849. Species algarum. Leipzig, Brockhaus, 922 p.

LAGERHEIM, G. 1887. Zur Entwickelungsgleschichte einiger Confervaceen. Ber. dt. Bot. Ges., Berlin, 5: 409-417.

MENEZES, M. 1986. Ficoflórula da Chapada dos Guimarães e arredores, Mato Grosso, Brasil: Euglenaceae pigmentadas (Euglenophyceae). Rickia, São Paulo, 13: 87-95.

MENEZES, M. \& FERNANDES, V.O. 1987. Euglenaceae pigmentadas do município de Cáceres e arredores, Mato Grosso, Brasil: uma contribuição ao seu conhecimento. Rickia, São Paulo, 14: 53-71.

PEREIRA, I.A. \& PARRA, O.O. 1984. Algas filamentosas dulceacüícolas de Chile. I. Algas bentónicas de Concepción. Gayana Bot., Concepción, 41 (3): 141-200.

PRESCOTT, G.W. 1975. Algae of the Western Great Lakes area. Iowa, 977 p.

RAMANATHAN, K.R. 1964. Ulotrichales. New Delhi, India Council of Agricultural Research, $188 \mathrm{p}$.

ROY, JJ. 1884. In COOKE, M.C. British fresh-water algae, exclusive of Desmidiae and Diatome, London, 329 p.

SCHMIDLE, W. 1901. Algen aus Brasilien. Hedwigia, Dresden, 40 (1): 45-54.

TRANSEAU, E.N. 1934. The genera of the Zygnemataceae. Trans. Am. microsc. Soc., Lancaster, 53: 201-208.

\section{LEGENDAS DAS FIGURAS}

FIGURA 1 - Mapa com indicação do estado de Mato Grosso e dos municípios de coleta: 1) Cáceres: 2) Mirassol d'Oeste: 3) Rio Branco; 4) Quatro Marcos; 5) Barra do Bugres; 6) Salto do Céu.

FIGURA 2 - Mougeotia abnormis - filamentos em conjugação escalariforme.

FIGURAS 3-4 - Spirogyra fennica - 3) detalhe das células férteis infladas; 4) aspecto geral do filamento vegetativo.

FIGURAS 5-6 - Spirogyra minutifossa - 5) filamentos em conjugação escalariforme; 6) detalhe da decoração dos zigósporos.

FIGURA 7 - Spirogyra neglecta - filamentos em conjugação escalariforme.

FIGURAS 8-9 - Zygnema insigne - 8) aspecto geral do filamento vegetativo: 9) detalhes da conjugação escalariforme.

FIGURA 10-11 - Zygnema oveidanum - 10) filamentos em conjugação escalariforme; 11) detalhe da decoração dos zigósporos.

FIGURA 12 - Oedogonium reinschii - aspecto geral do filamento.

FIGURAS 13-16 - Microspora tumidula - 13 e 16) aplanósporos; 14) formação dos aplanósporos: 15) detalhe do cloroplastídio e das peças em $\mathrm{H}$.

FIGURA 17-20 - Microspora willeana - 17) aspecto geral do filamento; 18) aplanósporos: 19) peças em $\mathrm{H} ; 20$ ) acinetos, cloroplastídios condensados.

FIGURAS 21-22 - Stigeoclonium farctum - 21) sistema ereto, ápices dos ramos; 22) sistema basal.

FIGURAS 23-24 - Stigeoclonium tenue - 23) sistema ereto, células longas e curtas; 24) ápices dos ramos.

FIGURAS 25-26 - Schizomeris leibleinii - 25) região distal do talo; 26 ) regiảo basal do talo. 

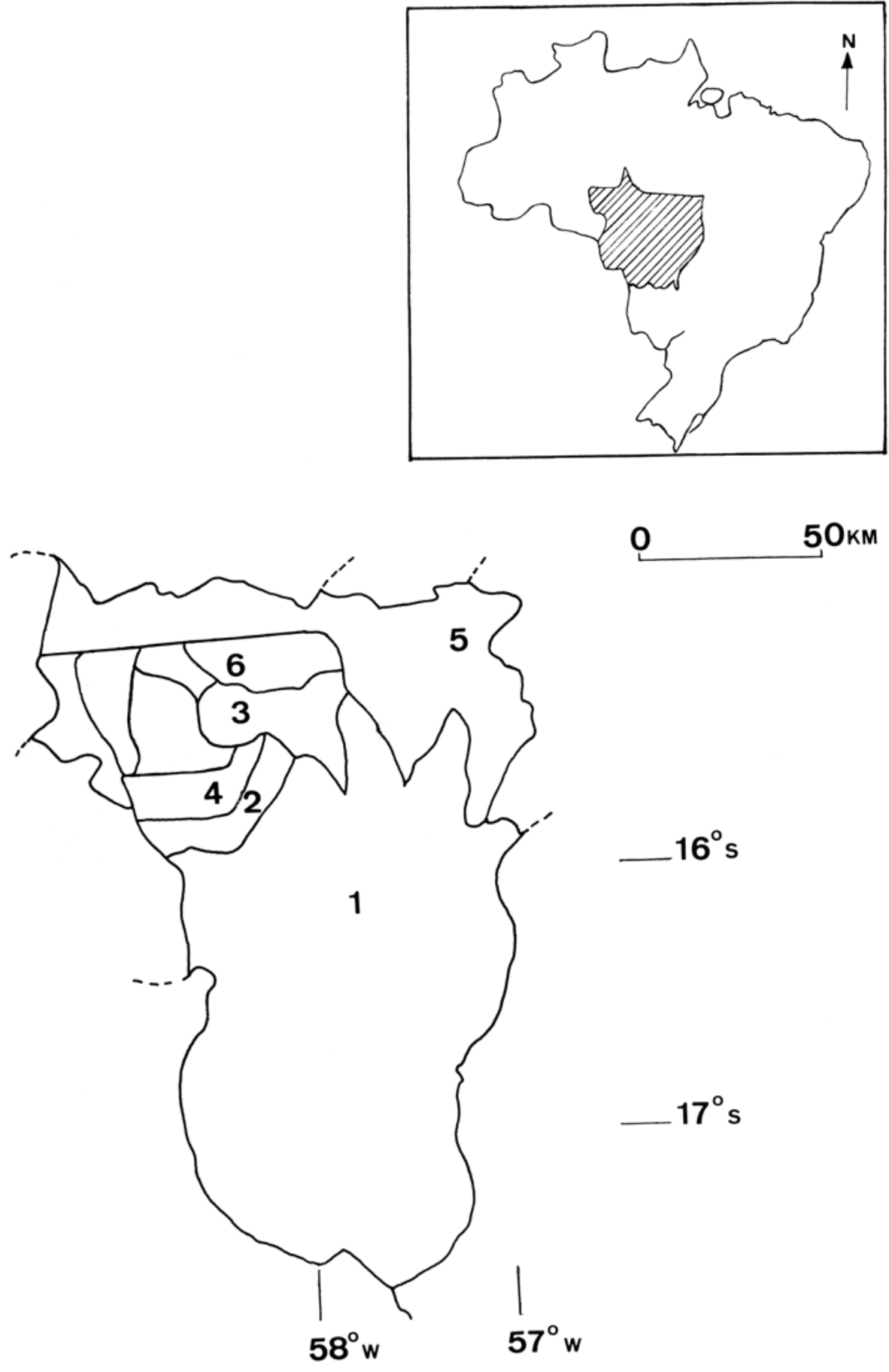


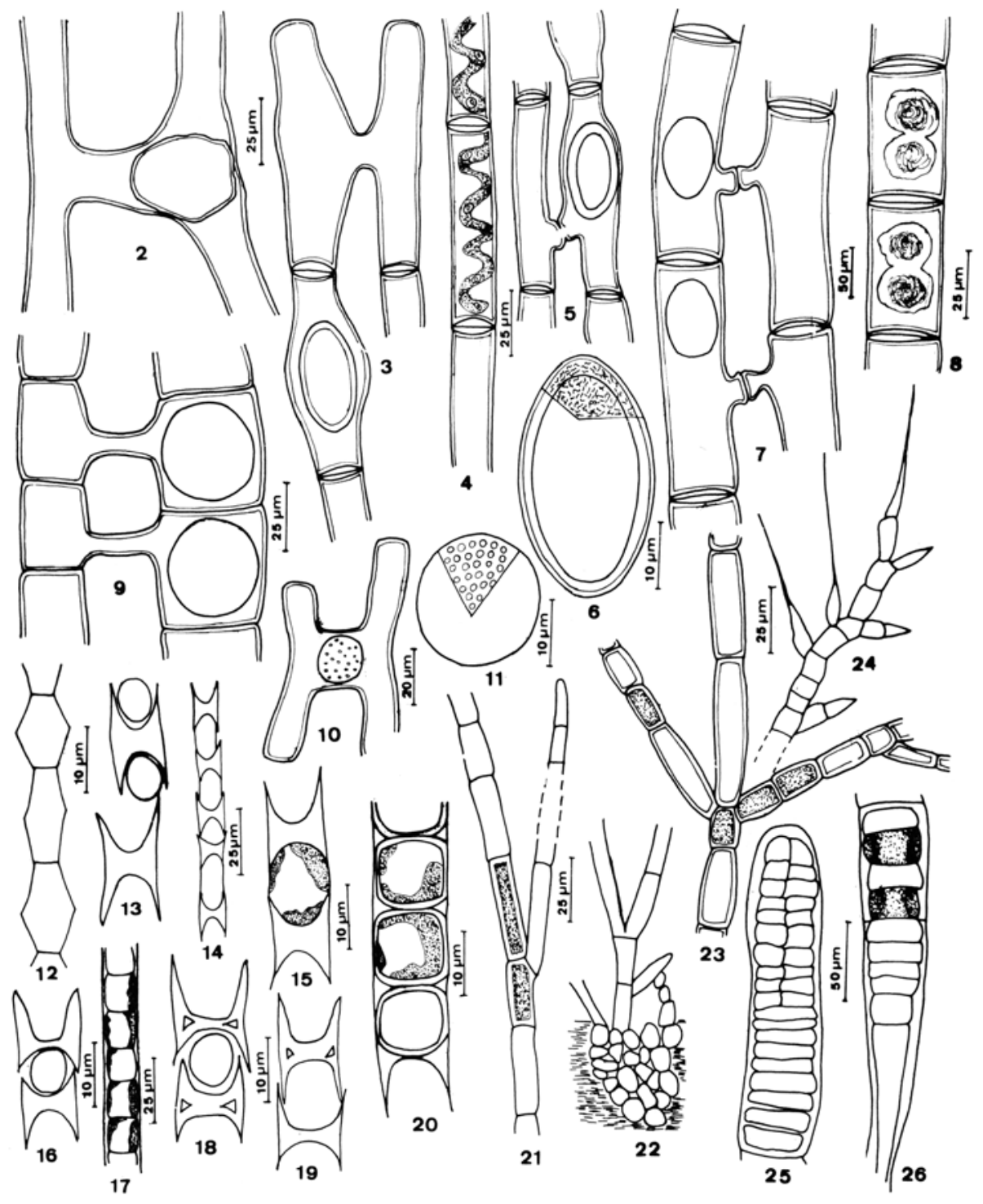

